\title{
High Speed Accurate Quantification with X-Max Large Area Silicon Drift Detectors
}

\author{
S. R. Burgess*, C.L. Collins*, J. Holland*, N. Rowlands** \\ *Oxford Instruments NanoAnalysis, Halifax Road, High Wycombe, HP12 3SE, UK \\ **Oxford Instruments NanoAnalysis, 300 Baker Avenue, Concord, Massachusetts, USA
}

Energy Dispersive Spectrometry (EDS) has been used for many years to analyse the chemical composition of materials. The introduction of silicon drift detectors (SDD) promises the potential of EDS analysis at acquisition rates in excess of 100,000cps, and therefore new opportunities for data collection times measured in seconds rather than minutes.

SDD detectors collect spectra with better resolution at 50,000cps than the best $\mathrm{Si}(\mathrm{Li})$ detectors working at an analytical count rate of 5,000cps. However, to achieve this 10 times speed improvement requires either a 10 times increase in beam current, or a 10 times increase in solid angle. For some micro-scale applications, on stable inorganic samples, a large increase in beam current is acceptable to exploit the speed benefits of SDD. However, for many applications, where small probe sizes and low beam current are important to maintain image quality, spatial resolution, and to minimise sample damage and contamination, this analysis strategy is not practical.

The introduction of very large area SDD detectors offers the capability of increasing count rates with little or no change in SEM collection conditions. It is important to assess whether the analysis results that are achieved under these high count rate conditions not only offer excellent spectral resolution, but also the same level of accuracy that can be achieved in practice using $\mathrm{Si}(\mathrm{Li})$ technology [1,2]. Otherwise any improvement in speed may come at the cost of inaccurate information about the sample being measured.

System design is critical to achieve these levels of speed and accuracy. For example at very high count rates there is increasing probability that two or more X-ray photons will be measured within the event resolving time of the electronics, the result being pulse pile-up [3]. The system electronics also need to maintain data fidelity so peak resolution, position and shape is maintained at all count rates in order for software algorithms to correctly identify element lines and measure the peak areas for each constituent element. This is particularly important when deconvolving overlapped X-ray peaks, where small changes in peak shape can lead to large changes in the calculated results.

To assess performance, tests were carried out using an $80 \mathrm{~mm}^{2} \mathrm{X}$-Max detector to determine the accuracy of results at different count rates. Spectra were collected at count rates up to 200,000cps on the mineral orthoclase. Fig 1. shows a spectrum collected from Orthoclase at 200,000cps using a live time of $2 \mathrm{~s}$ and a beam current of $4 \mathrm{nA}$, that demonstrates some problems with pile-up peaks at very high count rates. These peaks can give rise to false element identifications, but in addition each peak looses a proportion of counts based on the count rate. The loss of counts will give rise to low analytical totals when conventional standards analysis is used. However, even with standardless analysis using normalisation to $100 \%$, the application of pulse pile-up correction is still important for accurate results. In Table 1 results include some falsely identified peaks which 
alter the results for the correctly identified elements. In Table 2, even though false IDs are excluded, systematic errors appear at the higher rates. Only when pile up correction is included in Table 3 do the results remain close to expected values even at the highest rates.

Therefore the latest large area SDDs, combined with appropriate system design, offer virtually instant EDS analysis using operating conditions that are realistic for most SEMs and achieve accuracies comparable to those published using the best $\mathrm{Si}(\mathrm{Li})$ based technology. The potential productivity increases offered, in the order of 10 to 50 times, are clearly significant and can be achieved in practice on many more types of sample. Such increases are beneficial in all applications but are particularly significant where large amounts of quantitative data are required.

[1] S. Burgess et al, Microsc. Microanal, 13 (2007) 1432-1433

[2] D. Newbury, Microsc. Microanal. 4 (1999) 585 - 597

[3] P. Statham, Microchimica Acta, 155 (2006) 289-294

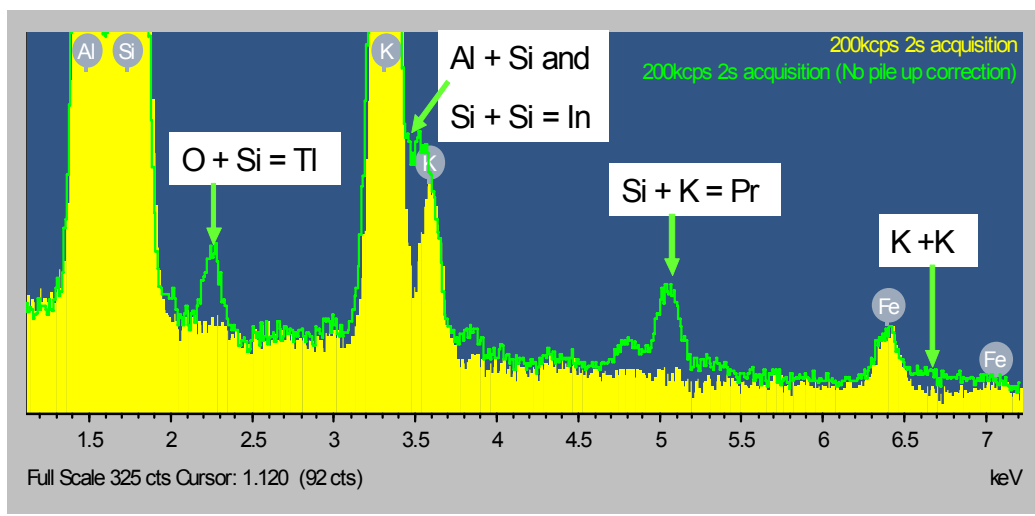

Figure 1. Spectrum of orthoclase collected using an X-Max $80 \mathrm{~mm}^{2}$ SDD at $200 \mathrm{kcps}$ in 2 seconds. Yellow shows the pile-up corrected spectrum. The green overlay shows the original uncorrected spectrum.

Table 1. Quantitative results of spectra collected at 5, 50, 100 and 200kcps. Results use standardless procedures, calculating oxygen by stoichiometry. Results include pile-up peaks incorrectly identified as In ( $\mathrm{Al}+\mathrm{Si}$ and $\mathrm{Si}+\mathrm{Si}), \operatorname{Pr}(\mathrm{Si}+\mathrm{K})$ and $\mathrm{Tl}(\mathrm{O}+\mathrm{Si})$

\begin{tabular}{|l|r|r|r|r|r|r|r|r|r|}
\hline & \multicolumn{1}{|l|}{$\mathrm{Na}$} & \multicolumn{1}{l|}{$\mathrm{Al}$} & \multicolumn{1}{l|}{$\mathrm{Si}$} & $\mathrm{K}$ & $\mathrm{Fe}$ & $\mathrm{In}$ & $\mathrm{Pr}$ & $\mathrm{TI}$ & $\mathrm{O}$ \\
\hline 200kcps 1s & 0.86 & 8.15 & 28.99 & 9.59 & 1.00 & 4.45 & 1.39 & 1.52 & 44.05 \\
\hline 150kcps 2s & 0.83 & 8.24 & 29.61 & 10.28 & 1.13 & 3.18 & 0.97 & 1.11 & 44.65 \\
\hline 50kcps 6s & 0.77 & 8.10 & 30.57 & 11.45 & 1.26 & 1.48 & 0.49 & 0.47 & 45.42 \\
\hline 5kcps 60s & 0.74 & 8.22 & 30.93 & 12.17 & 1.28 & 0.77 & 0.02 & 0.05 & 45.83 \\
\hline
\end{tabular}

\begin{tabular}{|c|c|c|c|c|c|c|}
\hline & $\mathrm{Na}$ & $\mathrm{Al}$ & Si & $\mathrm{K}$ & $\mathrm{Fe}$ & 0 \\
\hline $200 \mathrm{kcps} 1 \mathrm{~s}$ & 0.85 & 8.61 & 31.33 & 11.71 & 1.13 & 46.37 \\
\hline 150kcps 2s & 0.83 & 8.57 & 31.28 & 11.79 & 1.23 & 46.31 \\
\hline $50 \mathrm{kcps} 6 \mathrm{~s}$ & 0.76 & 8.25 & 31.36 & 12.14 & 1.31 & 46.18 \\
\hline $5 \mathrm{kcps} 60 \mathrm{~s}$ & 0.73 & 8.27 & 31.19 & 12.45 & 1.29 & 46.06 \\
\hline
\end{tabular}

Table 2. Results recalculated with the elements In, Pr and Tl, which are the result of pile-up artefacts, removed

\begin{tabular}{|l|r|r|r|r|r|r|}
\hline & \multicolumn{1}{|l|}{ Na } & \multicolumn{1}{l|}{ Al } & \multicolumn{1}{l|}{ Si } & \multicolumn{1}{l|}{ K } & \multicolumn{1}{l|}{ Fe } & \multicolumn{1}{l|}{ O } \\
\hline 200kcps 1s & 0.72 & 8.51 & 30.97 & 12.46 & 1.31 & 46.03 \\
\hline 150kcps 2s & 0.73 & 8.49 & 31.02 & 12.35 & 1.35 & 46.06 \\
\hline $50 \mathrm{kcps} 6 \mathrm{~s}$ & 0.73 & 8.23 & 31.26 & 12.36 & 1.33 & 46.09 \\
\hline $5 \mathrm{kcps} 60 \mathrm{~s}$ & 0.73 & 8.27 & 31.18 & 12.48 & 1.29 & 46.05 \\
\hline
\end{tabular}

Table 3. Results recalculated using the pileup correction algorithm 